\title{
Mit atrialer und ventrikulärer Arrhythmie assoziierte Faktoren bei stabilen Patienten mit chronisch- obstruktiver Lungenerkrankung
}

\author{
Schlüsselwörter \\ Chronisch obstruktive Lungenerkrankung · Arrhythmie \\ Bronchodilatator · Mehrdimensionale Untersuchungen
}

\begin{abstract}
Zusammenfassung
Hintergrund: Supraventrikuläre und ventrikuläre vorzeitige Komplexe (SVPC und VPC) sind mit chronisch obstruktiver Lungenerkrankung (COPD) und erhöhter Mortalität bei COPD-Patienten assoziiert. Jedoch liegen erst wenige Berichte zu den Ursachen von Arrhythmie bei COPD-Patienten vor.
\end{abstract}

Ziele: In dieser Studie untersuchten wir die Zusammenhänge zwischen kardiopulmonaler Dysfunktion und COPD, indem wir anhand von 24-stündigen EKG-Aufzeichnungen Patienten mit und ohne definierte Arrhythmie (>100 Schläge pro 24 h) verglichen.

Methoden: Patienten mit Arrhythmien durchliefen ein 24-h-EKG mit nachfolgender Lungenfunktionsprüfung, CT, EKG, Sechs-Minuten-Gehtest (6MGT), und BODE-Index-Berechnung (Body-MassIndex, Atemwegsobstruktion, modifizierte Medical Research Council Dyspnoea Scale, Belastungskapazität).

Ergebnisse: Von 103 Studienteilnehmern (71 COPD-Patienten und 32 Risikopatienten) lag bei 36 VPC vor, bei 45 SVPC, bei 20 beides und bei 42 keins von beidem. Der Sollwert der Einsekundenkapazität nach Bronchodilatatorgabe, der Anteil der Bereiche mit abgeschwächter Röntgendichte im CT sowie der BODE-Index waren in den SVPC- und VPC-Gruppen signifikant schlechter als in den Bezugsgruppen. Bei den Patienten in der VPC-Gruppe waren im Vergleich zur Bezugsgruppe ein signifikant erhöhter rechtsventrikulärer Druck und verstärkte Desaturierung beim 6MGT zu verzeichnen. In den multivariaten Analysen erwies sich in der SVPC-Gruppe die Bronchodilatation als signifikanter Risikofaktor, während in der VPC-Gruppe alle Parameter des BODE-Index mit Ausnahme des Dyspnoe-Score als Risikofaktoren identifiziert wurden.

Schlussfolgerungen: Vermehrtes Auftreten von SVPC könnte durch die Anwendung von Bronchodilatatoren verursacht werden, vermehrte VPC hingegen hängen wahrscheinlich mit der COPDeigenen Pathophysiologie zusammen.

(c) 2016 S. Karger GmbH, Freiburg

Originalartikel

\section{Atrial and Ventricular Arrhythmia-Associated Factors in Stable Patients with Chronic Obstructive Pulmonary Disease}

Yuji Kusunoki $^{\mathrm{a}, \mathrm{b}}$ Toshie Nakamura ${ }^{\mathrm{b}}$ Kumiko Hattori ${ }^{\mathrm{a}, \mathrm{b}}$ Takashi Motegi ${ }^{\mathrm{a}, \mathrm{b}}$ Takeo Ishii ${ }^{\mathrm{a}, \mathrm{b}}$ Akihiko Gemma Kozui Kida $^{\mathrm{a}, \mathrm{b}}$

${ }^{a}$ Respiratory Care Clinic;

bDepartment of Pulmonary Medicine, Infection, and Oncology, Nippon Medical School,

Tokyo, Japan 
Prof. Dr. Susanne Lang

susanne.lang@wkg.srh.de

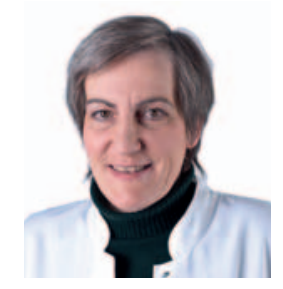

\section{Transfer in die Praxis}

In den letzten Jahren ist es zu einem Umdenken bei der Einteilung der COPD gekommen: weg von der Beurteilung des Schweregrads anhand der FEV1-Werte (Schweregrade I-IV) hin zu einem umfassenderen Ansatz (GOLD A-D), der neben den funktionellen Daten auch die Zahl der Exazerbationen, die subjektive Symptomlast und die Komorbiditäten erfasst. Neben den klassischen Phänotypen des Pink Puffer (Emphysemtyp), des Blue Bloater (Atemwegstyp) und dem Mischtyp (ACOS) sind nun auch Patienten mit zahlreichen Komorbiditäten im Fokus klinischer Untersuchungen. Der Begriff des «kardialen Phänotyps» der COPD ist von besonderer prognostischer Bedeutung, da Patienten mit COPD und kardiovaskulären Erkrankungen eine gemeinsame Risikogruppe darstellen können (Zigarettenrauchen). Zudem ist die Therapie der COPD mit LABA, LAMA und Theophyllin oder der Infektexazerbation mit Makrolidantibiotika mit typischen kardialen Nebenwirkungen assoziiert. Patienten mit COPD sind besonders gefährdet, am Rechtsherzversagen oder an gefährlichen Herzrhythmusstörungen zu versterben.

Die vorliegende prospektive, monozentrische Kohortenstudie aus Japan (103 Patienten) versuchte einen Zusammenhang zwischen dem Gebrauch von LABA, LAMA und Theophyllin, dem Schweregrad der COPD und dem Auftreten von Arrhythmien aufzuzeigen. Dazu wurden Patienten mit Arrhythmien (61 Patienten) im EKG-Screening eingeschlossen. Messparameter waren Lungenfunktion, BODE-Index, 24-h-EKG, Blutgase, Echokardiografie, CT-Morphologie und Labortests. Die Zahl der untersuchten Patienten war relativ gering, und nur 71 Patienten hatten COPD (32 Risikopatienten für die Entwicklung einer COPD). Die Mehrzahl der Patienten hatte einen geringen Schweregrad der COPD (Stadium IV; 3,9\%). Daher wurde nur 54,3\% ein LAMA und 64\% ein LABA-Präparat verabreicht. Der Anteil an Patienten mit Theophyllin lag mit 18,4\% ungewöhn- lich hoch. Insgesamt hatten 36 Patienten ventrikuläre Extrasystolen (VES), 45 Patienten supraventrikuläre Extrasystolen (SVES) und 20 Patienten beides. Wenn man die kleine Gruppe mit COPD im Stadium IV betrachtet, hatten alle Patienten SVES und VES. Die antiobstruktive Medikation führte zu signifikant mehr SVES, jedoch nicht VES.

Ein erhöhtes Auftreten von VES war mit dem BODE-Index, dem Schweregrad der COPD, schlechter Lungenfunktion, Desaturierung im Sechs-Minuten-Gehtest und Ausdehnung der überblähten Areale im CT korreliert. Die Autoren folgern, dass der Einsatz von LABA- oder LAMA Präparaten das Risiko fataler Arrythmien erhöht und gerade bei niedrigem Schweregrad der COPD oder bei Asthmapatienten kritisch überdacht werden sollte. Ein Langzeit-EGK wird als Screening empfohlen.

\section{Fazit}

Die sehr kleine Gruppe an Patienten mit überwiegend geringem bis mittlerem Schweregrad der COPD ist nicht geeignet, um den Zusammenhang zwischen klinisch signifikanten Arrhythmien und den eingesetzten Medikamenten zu belegen, da die Autoren nur die Anzahl an SVES und VES erfassten, aber keine Angaben zu tödlichen Ereignissen machen konnten, die durch Arrhythmien getriggert wurden. Metanalysen und Langzeitverlaufsstudien, die die Sicherheit von LABA-/ LAMA Kombinationen bei COPD Patienten untersuchten, ergaben keine erhöhte Sterblichkeit.

Dennoch sollte man in den Anfangsstadien der COPD die duale Bronchodilatation nicht unkritisch einsetzen, sondern das kardiale Risiko abwägen.

Kontaktadresse: Prof. Dr. Susanne Lang, SRH Wald-Klinikum Gera, Medizinische Klinik 2: Klinik für Hämatologie und Onkologie, Pneumologie und Infektologie, Akutgeriatrie und Diabetologie, Straße des Friedens 122, 07548 Gera, Deutschland 